\title{
Multiresistente Tb soll ausgerottet werden - aber wie?
}

Deutschland ist schon lange Drehscheibe zwischen Ost und West für multiresistente Tuberkulose, und die Situation dürfte sich im Gefolge der Flüchtlingskrise verschärfen. Zugleich planen WHO und ERS die Ausrottung der Tb. Ihre Agenda wurde auf dem ERS-Kongress vorgestellt.

„Ein einziger Patient kann einen resistenten Tb-Stamm durch die halbe Welt tragen", betonte Dr. Marieke van der Werf, European Centre for Disease Control and Prevention, Stockholm. Derzeit geht man von etwa neun Millionen Tuberkulosepatienten weltweit aus, von denen ca. 300.000 eine M/XDR-Tb haben.

\section{Hohe M/XDR-Raten in China und Russland}

Zurzeit liegen Resistenz-Hotspots in den Nachfolgestaaten der UdSSR und in China, woher der besonders weit verbreitete multiresistente Tb-Stamm Beijing stammt, der in Europa für die größten Ausbrüche verantwortlich ist.

Solange Tuberkulosepatienten in viele Ländern keine adäquate Therapie erhalten, wird sich daran wohl wenig ändern, denn MDR (multiresistente)- und XDR (auch gegen Zweitlinien-Tuberkulostatika resistente)-Stämme können jederzeit und überall entstehen. Immerhin: Zurzeit scheint das Problem aus europäischer Sicht nicht zuzunehmen - was jedoch jederzeit umschlagen kann.

Weltgesundheitsorganisation und European Respiratory Society (ERS) haben sich die Ausrottung der Tuberkulose auf die Fahnen geschrieben und soeben eine „Agenda For Action“ verabschiedet, mit der die Erreichung dieses Ziels beschleunigt werden soll. Unverzichtbare Voraussetzungen: Die Erkennung und Behandlung aller infizierten Patienten und die Prävention neuer Infektionen.

\section{Digitale Strategien für die Eradikation}

Ein zentraler Ansatz liegt in der Integration digitaler Medien in die bereits bestehenden WHO-Strategien. „Informations- und Kommunikationstechnologie sind starke Werkzeuge, die uns dabei helfen werden, unsere ambitionierten neuen Ziele auch in Regionen zu erreichen, die über wenig finanzielle Ressourcen verfügen", meinte Dr. Mario Raviglione, Direktor des globalen WHO-Tuberkulose-Programms.

Die M/XDR-Stämme komplizieren die Eradikationspläne, aber prinzipiell sind auch multiresistente Tuberkulosen zu einem hohen Prozentsatz heilbar: „Die Erfolgsraten liegen bei 70 bis 80 Prozent", sagte Prof. Christoph Lange, Leiter des Klinischen Tuberkulosezentrums am Forschungszentrum Borstel in Lübeck. Immerhin stehen heute fast 30 Antibiotika verschiedener Klassen zur Verfügung, mit denen die $\mathrm{Tb}$ angegangen werden kann.

Der Aufwand ist allerdings hoch, denn „ein Patient, bei dem heute, Ende September 2015, eine M/XDR-Tuberkulose diagnostiziert und die Behandlung begonnen wird, muss bis Mai 2017 durchhalten", so Lange.

Um eine MDR-Tb zu behandeln, werden mindestens vier, für eine XDR-Tb fünf Wirkstoffe kombiniert - das geht auch ins Geld. Um einen „normalen“ Tb-Patienten zu heilen, werden maximal 1.300 Euro benötigt - bei MDR-Tb sind es fast 80.000 Euro, bei XDR sogar fast doppelt so viel. Engmaschige Patientenführung stellt dabei einen zentralen Erfolgsfaktor dar. Manuela Arand 